\title{
Invasive prenatal testing at a Tertiary Fetal Medicine referral center in Sri Lanka: A service evaluation audit
}

\author{
Padeniya AGPM ${ }^{1}$, Dias TD ${ }^{1}$
}

\section{INTRODUCTION}

Genetics is being increasingly used in diagnosis as well as in treatment in a wide range of areas of Reproductive Medicine. Prenatal genetic testing is a key area that is gaining popularity in many settings including Sri Lanka. This involves a multidisciplinary approach with fetal medicine and clinical genetics.

All pregnancies carry a baseline risk of $3-4 \%$ to any type of birth defect ${ }^{1}$. The severity of the anomaly may vary, reflecting the wide spectrum of mutations. Such defects can be due to inherited genetic abnormalities, de novo mutations arising in the gametes or the embryo, or effects of environmental influences that occur during pregnancy ${ }^{1,2}$. Invasive prenatal testing includes the genetic evaluation of the fetus/embryo by an invasive method before birth. This requires collection of samples of placental or fetal cells, either by chorionic villous sampling (CVS) or amniocentesis, collection of exfoliated fetal cells, or direct fetal blood sampling through cordocentesis $^{1,3,4}$. An increased risk, above the background risk, of fetal chromosomal aneuploidy is the most common indication for invasive prenatal testing. The contributory causes for such an increased risk calculation include the maternal age of over 35 years, a previous child with de novo chromosome abnormality, family history of detectable genetic defect, parental consanguinity in families with recessive diseases, maternal systemic illness, teratogen

Fetal Medicine Unit, Ninewells Care Hospital, Colombo, Sri Lanka

Correspondence: Dr. Tiran Dias

E-mail: thiran_dias@yahoo.com

Competing interests: None exposure including medication and abnormalities in amniotic fluid volume ${ }^{5}$.

Prenatal invasive testing poses certain procedure related risks. Spontaneous miscarriage associated with CVS and amniocentesis is estimated to be around $1-2 \%{ }^{2}$, whereas cordocentesis is associated with an increased risk of fetal loss ranging from 1.5-2.8\% A recent study suggested the risk of miscarriage with invasive testing to be much lower ${ }^{7}$. It is known that $1-2 \%$ of women will miscarry during early 2 nd trimester, even if they have not undergone any invasive testing ${ }^{7}$. Therefore, it is extremely difficult to determine if a miscarriage that follows an invasive test is directly related to the procedure ${ }^{7}$. In clinical practice, a spontaneous miscarriage that occurs within 10 days of the invasive procedure is considered to be procedure related. The skill of the operator will influence both the success rate of the procedure as well as the procedure-related miscarriage rate8. Operators who perform a small number of procedures per year may have an failure rate and an increased rate of procedure-related pregnancy loss8. Confined placental mosaicism seen with CVS may also produce false results ${ }^{2,9}$. Some authorities have recommended a minimum of 30 ultrasound guided invasive procedures per annum to maintain the competency $^{8}$. Other indicators of quality of services include the rate of infection and maternal cell contamination.

It is recommended to carryout regular clinical audits on the outcome of invasive procedures in order to monitor and maintain the quality of care. We conducted an audit at our center assessing clinical indications, the methods of testing, and the shortterm outcome.

\section{METHODS}

A retrospective audit was carried out on all invasive prenatal tests done between 2013 July and 2014 July at the Fetal Medicine Unit. All the invasive procedures were done by a Fetal Medicine Foundation United Kingdom accredited operator ${ }^{11}$. Patient information and type of invasive procedure was obtained from the Fetal Medicine Unit database and Fetal Medicine Foundation first trimester screening software. As a good practice the outcome data is regularly collected and saved in a purpose built data sheet at this unit. The outcome data audited included results of the tests, the infection rate and procedure related miscarriage (within 10days of procedure) rate.

\section{RESULTS}

A total of 76 patients underwent prenatal testing during the period audited (table). This includes 13 cordocentesis procedures and 63 amniocentesis procedures (Figure 1). There were 10 patients who were lost to follow-up.

Screening for common beta thalassaemia mutations was performed in five samples and four were found to be beta thalassaemia carriers for IVSI-V $(\mathrm{G}>\mathrm{C})$, IVSI-I $(\mathrm{G}>\mathrm{A})$ and $C D 26(G>A)$ mutations while one had a normal genotype. Therapeutic amniocentesis (amnioreduction) was performed in three $(3.9 \%)$ patients for polyhydramnios. One cordocentesis was performed for flow cytometry in a woman with a positive family history of severe combined immunodeficiency disease (SCID). Vesico-amniotic shunting was done in a fetus with an obstructive uropathy.

There were no spontaneous miscarriages reported in chromosomally and structurally normal fetuses. A total of three miscarriages were noted during 


\begin{tabular}{|l|c|c|}
\hline \multicolumn{3}{|c|}{ Table1: Description of the study population } \\
\hline & Mean (range) & SD \\
\hline Maternal age & $\begin{array}{c}32.7 \\
(21-46)\end{array}$ & 6.4 \\
\hline Period of gestation (weeks) at Amniocentesis & 18 & 4.5 \\
\hline Period of gestation (weeks) at Cordocentesis & $(15-34)$ & 4.6 \\
\hline
\end{tabular}

\section{Figure 1: Type of invasive prenatal testing and their frequency}

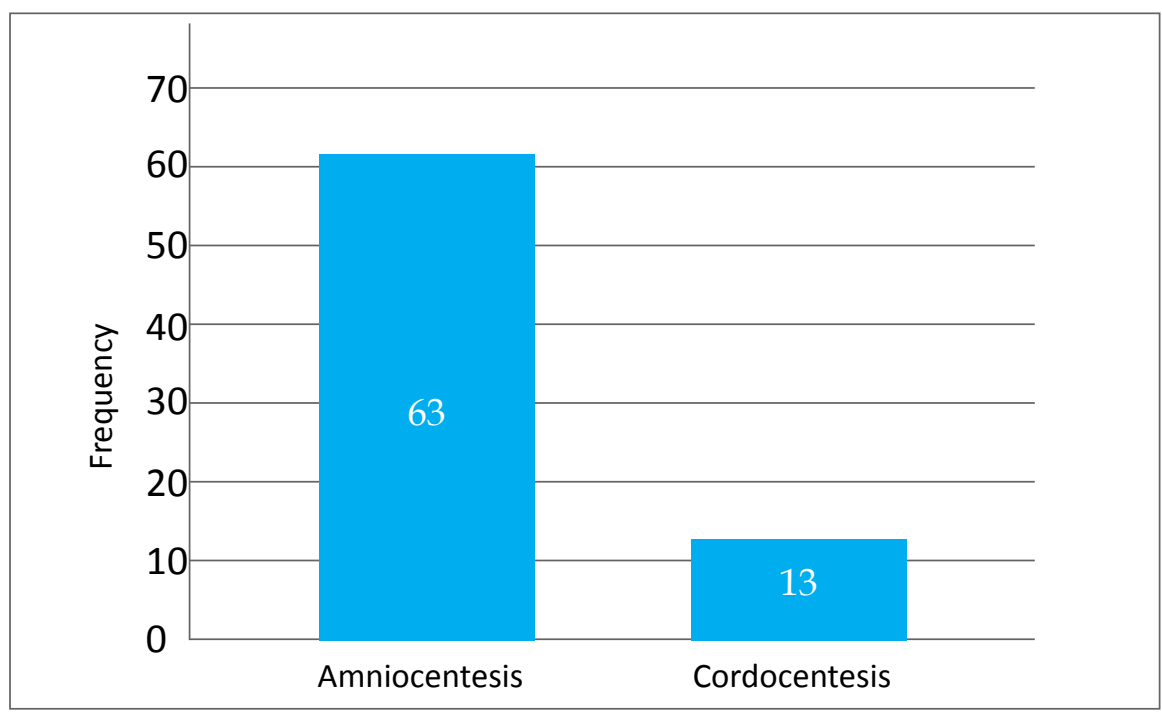

Figure 2: The distribution of the different types of chromosomal abnormalities detected during the prenatal testing

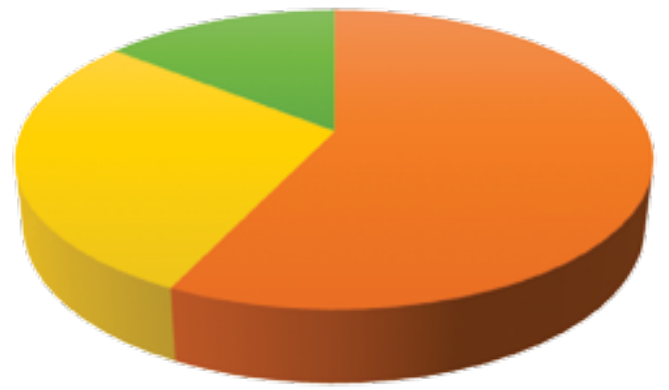

Trisomy21 Trisomy18 Klinefelter syndrome the audit period. Chromosomal abnormalities were detected in $7(9.2 \%)$ patients and distribution of abnormalities are shown in figure 2. All of them had either a chromosomal or structure anomaly (Trisomy 18, non-immune hydrops and multiple abnormalities in one each). None of the patients developed procedure related infection or other complications following invasive prenatal testing.

\section{DISCUSSION}

Amniocentesis is the most common invasive prenatal diagnostic procedure undertaken at the unit and this is comparable with the trend worldwide. Regular audit should be performed in order to maintain quality care in prenatal Medicine. The procedure related complications were par??? with recommended standards. This indicates adequate training and maintenance of skills of the operator as occasional operators who perform a lower number of procedures per year may have increased rates of procedure-related complications.

\section{CONCLUSION:}

Our data is comparable with the available international standards. We did not have procedure related miscarriages in healthy fetuses and no other procedure related complications were seen. This audit demonstrated that the procedure related complications of the unit are low among chromosomally and structurally normal fetuses after invasive prenatal testing, thus conforming acceptable safety standards

\section{REFERENCES}

1. Bodurtha, J. and J.F. Strauss, 3rd, Genomics and perinatal care. N Engl J Med, 2012. 366(1): p. 64-73.

2. Brezina, P.R. and W.G. Kearns, The evolving role of genetics in reproductive medicine. Obstet Gynecol Clin North Am, 2014. 41(1): p. 41-55.

3. Mendez-Rosado, L.A., et al., Antenatal cytogenetic testing in Havana, Cuba. MEDICC Rev, 2014. 16(3-4): p. 27-34.

4. Karnpean, R., Fetal blood sampling in 
prenatal diagnosis of thalassemia at late pregnancy. J Med Assoc Thai, 2014. 97 Suppl 4: p. S49-55.

5. Sadlecki, P., et al., [Indications for genetic amniocentesis investigated at the Department of Gynecology, Obstetrics, and Oncologic Gynecology, Nicolaus Copernicus University, Collegium Medicum, Bydgoszcz]. Ginekol Pol, 2014. 85(6): p. 420-3.

6. Tongsong, T., et al., Second-trimester cordocentesis and the risk of small for gestational age and preterm birth. Obstet Gynecol, 2014. 124(5): p. 919-25.
7. Akolekar R, Beta J, Picciarelli G, Ogilvie $\mathrm{C}$, D'Antonio F. Procedure-related risk of miscarriage following amniocentesis and chorionic villus sampling: a systematic review and meta-analysis. Ultrasound Obstet Gynecol. 2015 Jan;45(1):16-26. doi: 10.1002/uog. 14636.

8. Royal College of Obstetricians and Gynaecologists. Amniocentesis and Chorionic Villus Sampling. Green-top Guideline No. 8 June 2010

9. Pergament, E. and D. Pergament, Reproductive decisions after fetal genetic counselling. Best Pract Res Clin Obstet
Gynaecol, 2012. 26(5): p. 517-29.

10. Fisher, C.A., et al., The molecular basis for the thalassaemias in Sri Lanka. Br J Haematol, 2003. 121(4): p. 662-71.

11. Fetal Medicine Foundation accredited practitioners-Invasive procedures. Health care professionals who have obtained the Certificate of competence in Invasive procedures. https://fetalmedicine.org/ $\mathrm{map} /$ certified/7 